\title{
Graded magnonic index and spin wave Fano resonances in magnetic structures: Excite, direct, capture
}

\author{
V. V. Kruglyak, ${ }^{1, *}$ C. S. Davies,${ }^{1}$ Y. Au, ${ }^{1}$ F. B. Mushenok,${ }^{1}$ G. Hrkac,${ }^{1}$ N. J. Whitehead ${ }^{1}$ \\ S. A. R. Horsley, ${ }^{1}$ T. G. Philbin, ${ }^{1}$ V. D. Poimanov, ${ }^{2}$ R. Dost,${ }^{3}$ D. A. Allwood, ${ }^{3}$ \\ B. J. Inkson, ${ }^{3}$ and A. N. Kuchko ${ }^{4}$ \\ ${ }^{1}$ School of Physics, University of Exeter, Exeter, United Kingdom \\ ${ }^{2}$ Donetsk National University, Donetsk, Ukraine \\ ${ }^{3}$ Department of Materials Science and Engineering, University of \\ Sheffield, Sheffield, S1 3JD, United Kingdom \\ ${ }^{4}$ Institute of Magnetism of NAS of Ukraine, Kiev, Ukraine
}

\begin{abstract}
Starting from the general topic and fundamentals of magnonics, we discuss and provide demonstrations of exciting new physics and technological opportunities associated with the graded magnonic index and spin wave Fano resonances, highlighting them as the next big thing in magnonics research.
\end{abstract}

* Corresponding author: V.V.Kruglyak@exeter.ac.uk 


\section{Introduction}

Even the most general definition of magnonics, ${ }^{1,2}$ as the study of spin waves, ${ }^{3,4}$ leaves a lot of freedom for interpretation and scientific discussion of directions of the field's further development. Thus, we have recently seen a number of excellent review papers with emphasis on different aspects of spin wave research and technology, e.g. magnonic crystals and metamaterials, ${ }^{5-9}$ photo-magnonics, ${ }^{10,11}$ spin caloritronics, ${ }^{12}$ magnon spintronics, ${ }^{13-15}$ nanoscience, ${ }^{16-19}$ and applications of spin waves in microwave signal processing and data manipulation. ${ }^{20-22}$ There is however an aspect of magnonics that has been both ubiquitous and somewhat underrated so far: magnonics is the study not only of spin but also (and most importantly) of waves, which have an extremely rich and peculiar dispersion that is nonlinear, anisotropic and non-reciprocal. The spin wave dispersion is very sensitive to the sample's magnetic properties and micromagnetic state, including both the internal magnetic field and magnetisation, so that spin waves are rarely observed to propagate in uniform media. Inspired by and feeding from other fields of wave physics, such as quantum mechanics, ${ }^{23}$ graded-index optics ${ }^{24}$ and transformation optics, ${ }^{25}$ we have recently tried to formulate the concept of graded-index magnonics as a unifying theme focusing on general aspects of spin wave excitation and propagation in media with continuously non-uniform properties. ${ }^{26,27}$ Graded-index magnonics is the main topic of this Chapter, and is also highlighted throughout the rest of this Book.

The term "graded-index magnonics" implies existence of a single quantity, often dubbed magnonic or spin wave refractive index or more simply "magnonic index", that describes fully the spin wave dispersion in a (continuous and uniform) sample. Yet, it would be extremely difficult (if at all possible) to do this, given the plethora of factors that influence the (already complex) spin wave dispersion, with their sheer diversity necessarily limiting any definition to special cases and approximations. ${ }^{28-35}$ So, we treat the term "magnonic index" here as merely a tag encompassing the entirety of spin wave dispersion and its modifications dictated by the variation of the magnetic medium's properties.

With a historical perspective on graded-index magnonics already presented in Ref. 27, we limit coverage of this Chapter mostly to our own published and forthcoming results, which deal with the spatial variation of the magnonic index induced by patterning thin magnetic films of the same material (Permalloy). Such patterning has been extensively discussed in the context of spin wave confinement in the previous Book of this series. ${ }^{36}$ So, given the additional focus of this Book on spin wave propagation, this leads to another topic 
of central importance for this Chapter - spin wave Fano resonances. ${ }^{37}$ Resulting from interaction between systems with a discrete spectrum and continuous spectra, Fano resonances have been widely studied for other wave excitations ${ }^{38}$ but have been explored and exploited much less in magnonics. ${ }^{39,40}$ Here, we will demonstrate retrospectively their relevance to some of our previous observations.

It is conventional to discuss spin wave phenomena in the context of magnonic technology, focusing on data and / or microwave signal processing. The areal density of transistors in integrated circuits was famously conjectured by Moore ${ }^{41}$ in 1965 to double every two years as part of "Moore's Law". This growth has been consistently observed, and modern hand-held computing devices can easily contain gigabytes of memory and process data at $\mathrm{GHz}$ rates. However, in the most recent period, the transistor areal density took three years to double, while the clock rate of modern processers has not advanced since $2004 .{ }^{42,43}$ To safeguard the technological progress, condensed matter researchers are now striving to develop technologies that will enable computing devices to break past the barriers faced by electronics, with magnonics being one of such emerging alternatives. More generally, magnonics is envisioned to become a natural companion to electronic, spintronic and microwave technologies, which could offer additional functionalities (e.g. non-volatility and magnetic field tunability/programmability) to the more conventional technologies.

The place of magnonics among its sister fields of research and associated technologies is illustrated in Fig. 1 (a). Direct current (dc) electricity and conventional semiconductor electronics use the one-way translational motion of charge to transmit energy and information across a circuit, while the same goal is achieved using charge oscillations in ac electricity and electromagnetics. ${ }^{44}$ Each charged particle in an ensemble can experience a local electromagnetic oscillation relative to its individual equilibrium position. Collectively, these phase-coherent oscillations give rise to a net wave-like motion, which transmits energy (signal) without charge or particle transport. An immediate successor of electronics, spintronics, instead exploits the translational motion of spins. However, the use of the wave motion of spins to carry and process information has only just begun to be explored, giving rise to the recent burst in magnonics research. The relationship between spintronics and magnonics is analogous to that between dc electricity and electromagnetics, ${ }^{45}$ with an important difference that spin waves can transfer not only energy but also angular momentum. ${ }^{12-15}$ 
(a) translational motion
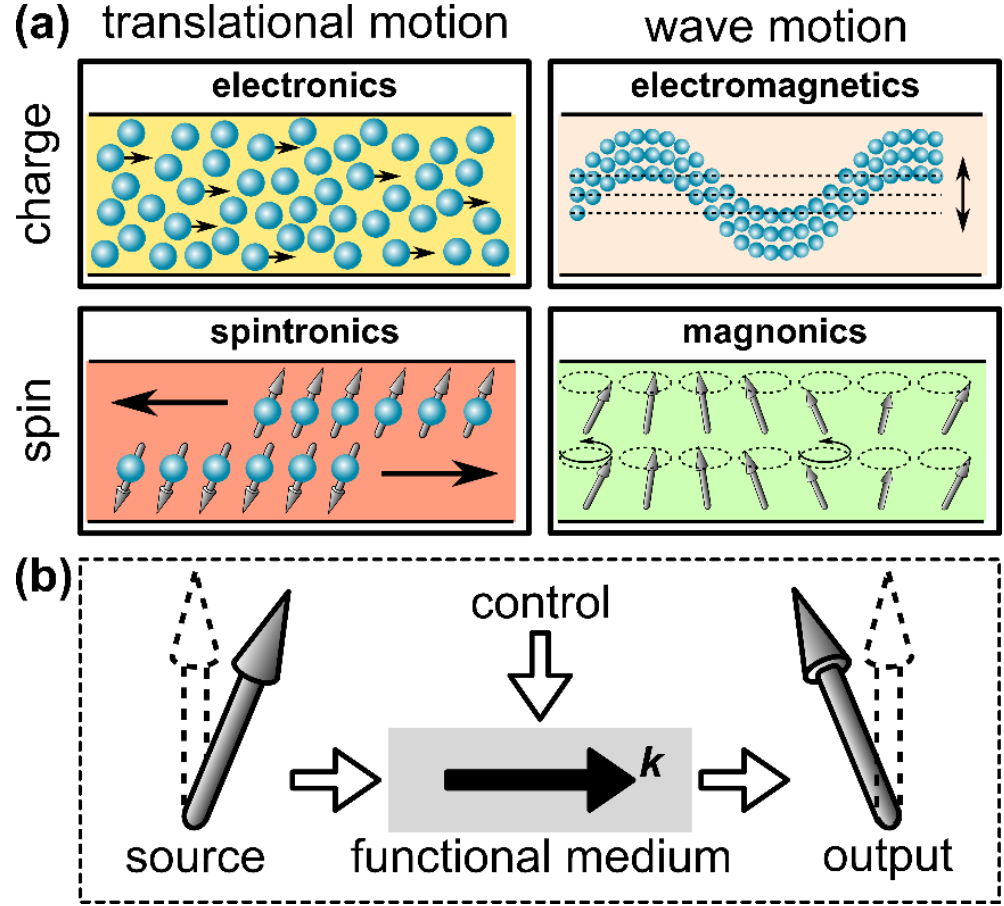

Fig. 1 (a) The relationship between magnonics and its sister fields of research and technology. (b) A block diagram of a generic magnonic device and its constituents. (After Ref. 46)

This Chapter is organised using the scheme that we introduced earlier in Ref. 1. Specifically, we consider the generic magnonic device shown in Fig. 1 (b). Then, even findings of a fundamental nature are convenient to discuss in terms of their relevance to one of the four main constituents of the device: source, functional medium, output and control (mechanism). We show that the concepts of graded magnonic index and spin wave Fano resonances are relevant to each of the constituents. In Section II, we remind the reader about the main aspects of the spin wave dispersion. In Sections III, IV and V, we discuss how the graded magnonic index and spin wave Fano resonances could be used to aid spin wave emission, steering and detection, respectively. In Section VI, we discuss spin wave control and show some conceptual device designs, for the sake of illustration.

\section{Spin wave dispersion}

Generally, magnetic systems contain ordered ensembles of tiny magnetic moments spins - coupled by the exchange interaction. On a quasi-classical basis, the magnetic moments are individually capable of precessing about their equilibrium orientation. Due to 
the coupling between spins, it is possible to excite phase-coherent precessional waves of magnetisation - the average magnetic moment per unit volume. It is these waves that are called "spin waves", and their quanta are called "magnons".,4 Like other waves, spin waves are characterised by their amplitude, phase, frequency, wave vector, and group and phase velocities, each representing a resource for signal manipulation.

The key feature that makes spin waves unique is their dispersion (Fig. 2 (a)), which can be peculiarly anisotropic depending on the dominant interaction between magnetic moments. ${ }^{3,4}$ There are two main interactions to consider. The quantum-mechanical exchange interaction (responsible for the magnetic ordering) dominates over nanometre wavelengths and gives rise to an isotropic, parabolic dispersion of so-called exchange spin waves. In the wavelength range from hundreds of nanometres and above, spin waves are said to be magnetostatic (or dipolar) in nature, since their dispersion is dominated by the anisotropic magneto-dipole interaction. In the intermediate wavelength range, when both exchange and dipolar energies contribute noticeably to the dispersion, the spin waves are said to be of dipole-exchange character. In either case, spin waves travel with typical speeds of several kilometres per second, and so are admittedly rather slow. However, this also means that, at the same frequency, they have a much shorter wavelength compared to e.g. electromagnetic waves, paving the way towards device miniaturisation.

The frequency of spin waves with infinite wavelength (and therefore also zero wave vector) is called ferromagnetic resonance $(\mathrm{FMR})$ frequency, $f_{\mathrm{FMR}}$, which corresponds to the magnon energy gap. An incident microwave at this frequency will couple to the magnetisation precession in the sample most strongly, exciting its uniform (fundamental) precessional mode. A constant magnetic field applied to the sample (i.e. bias magnetic field) shifts $f_{\mathrm{FMR}}$ up or down, along with the rest of the dispersion. The direction and speed of the energy transfer by spin waves is determined by their group velocity, defined as the gradient of the angular frequency in reciprocal space. In addition to the bias magnetic field, the spin wave group velocity depends dramatically on the angle between the spin wave wave vector and the magnetisation. In particular, the group velocity is negative (roughly antiparallel to the wave vector $\boldsymbol{k}$ ) for backward volume spin waves, i.e. spin waves with their frequencies below $f_{\mathrm{FMR}}$ and their wave vectors roughly parallel to the magnetisation. Yet, it is positive (roughly parallel to $\boldsymbol{k}$ ) for Damon-Eshbach spin waves, i.e. spin waves with their frequencies above $f_{\mathrm{FMR}}$ and their wave vectors roughly orthogonal to the magnetisation. In general, the 
direction of the group velocity is convenient to predict using isofrequency curves, i.e. curves of constant frequency. ${ }^{47}$ The group velocity is always orthogonal to the isofrequency curves in the reciprocal space, in the same way as the electric field is always orthogonal to the curves of constant potential in real space. The dispersion anisotropy is strongest for magnetostatic spin waves, quickly diminishing as the wavelength decreases.

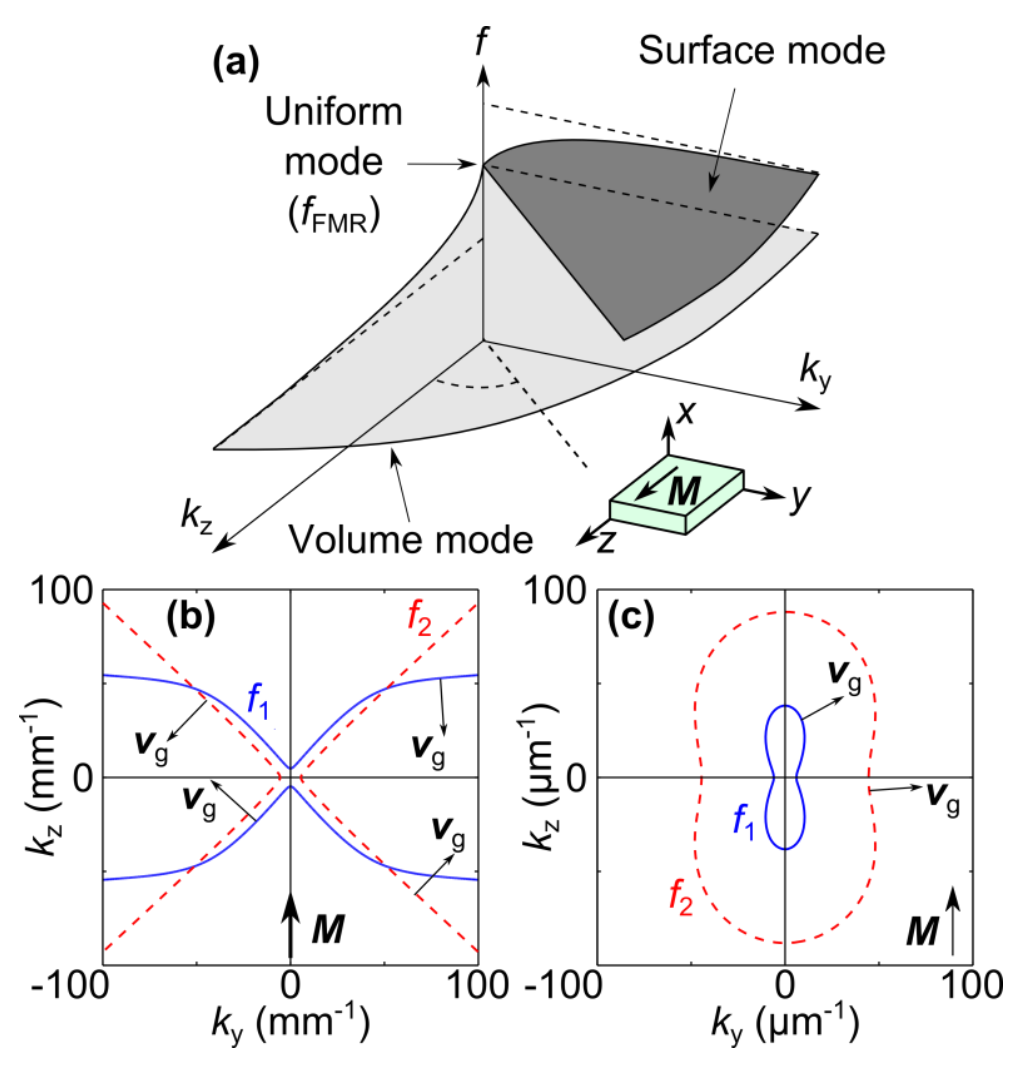

Fig. 2 (a) The magnetostatic spin wave dispersion plotted in one quadrant of reciprocal space for a film in the $y z$-plane with in-plane magnetisation in the $z$-direction. Above and below the FMR frequency $f_{\mathrm{FMR}}$, there is a single and infinite manifold of dispersion branches, referred to as the surface (Damon-Eshbach) mode and backward volume modes, respectively. Only the first branch of the volume spin wave modes is shown, corresponding to the uniform precession across the film thickness. (b) The isofrequency curves characterising the propagation of magnetostatic spin waves, where $f_{2}>f_{\mathrm{FMR}}>f_{1}$. (c) The isofrequency curves characterising the propagation of dipole-exchange spin waves, where $f_{2}>f_{1}>f_{\mathrm{FMR}}$.

The main attraction of the spin wave dispersion for wave physicists is the variety of factors that determine and therefore could be used to tailor its character. The compositional modulation of magnetic media represents the most obvious (albeit technologically 
challenging) way to achieve this. The dispersion of magnetostatic spin waves in thin-film magnetic samples is also sensitive to the variation of the film thickness. Moreover, the finite thickness of magnetic film structures leads to quantisation and thus appearance of several dispersion branches for spin waves (of any sort) propagating within the plane of the film. The same is true for the effect of the lateral quantisation in waveguides with a finite width. The dispersion is different for each branch and depends on the film thickness, which suggests the continuous variation of the thickness and / or width as a means by which to control the spin wave propagation in patterned magnetic structures.

The presence of lateral boundaries leads to a non-uniform demagnetising field and internal magnetic field (and therefore graded magnonic index) in their vicinity. ${ }^{36}$ The nonuniform internal magnetic field can lead to a non-uniform configuration of the magnetisation. Furthermore, the magnetisation is almost necessarily non-uniform in samples with a significant antisymmetric exchange interaction (Dzyaloshinskii-Moriya interaction) ${ }^{48,49}$ and those with spatially varying directions of the easy and / or hard magnetisation axes of the magnetic anisotropy. ${ }^{3,4}$ The field and magnetisation non-uniformity can also be delivered by a non-uniform bias magnetic field, which in addition offers an opportunity to study magnonic phenomena in time-varying graded-index magnonic landscapes. ${ }^{22}$ The non-uniform magnetisation textures modify the magnonic index not only through the dispersion anisotropy (Fig. 2) but also via modification of the demagnetising field ${ }^{36}$ and emergence of topological effects, such as Berry phase,$^{50}$ geometrical anisotropy ${ }^{51,52}$ and topological protection. ${ }^{53,54}$ The non-uniformity breaks the translational invariance, limiting the use of the wave vector and therefore the notion of wave dispersion. The notable exceptions are given by the periodic and slowly varying (spatially) non-uniformities, the former giving rise to magnonic crystals ${ }^{1,2,5-9}$ and the latter allowing the use of the geometrical optics (quasi-classical) approximation. ${ }^{26,28}$

\section{Spin wave excitation}

To excite propagating spin waves, it is necessary to perturb the magnetisation both quickly and locally, so that the frequency and wave vector of the spin wave to be excited are covered by the spectrum of the perturbation. Conventionally, this is done using microwave microstrips ${ }^{2,7}$ or spin-transfer torque techniques. ${ }^{13,14}$ Another promising mechanism of spin wave excitation involves coupling free-space microwaves waves to spin waves through the use of local magnetic inhomogeneities, which can have the form of either a graded magnonic 
index ${ }^{55-60}$ or Fano resonators. ${ }^{26,61-63}$ The main idea behind the method is that the spatial nonuniformity breaks the translational symmetry in the system, thereby enabling coupling between the microwave magnetic field and spin waves irrespective of their wavelengths.

In the most basic case, the FMR frequency of magnetic samples depends on the saturation magnetisation of the material, the sample's dimensions and the applied bias magnetic field. The same is true for spin wave (higher-order) resonances. It is possible to design a system of two neighbouring (or connecting) magnetic elements that have different dominant resonance frequencies for a given bias magnetic field value / orientation. When the entire system is pumped by a harmonic microwave magnetic field at the higher resonance frequency, the resonance is excited in one element only (the "transducer"). The coupling between the magnetisations of the two elements then leads to injection of spin waves into the second element (the "waveguide"), with their spin wave vector dictated by the magnonic dispersion in the waveguide. Such a resonance, in which the energy from one resonantly excited element with a discrete spectrum is "leaked" into wave modes propagating in the element with a continuous spectrum, is an example of a Fano resonance. ${ }^{37,38}$

This Fano resonance assisted mechanism of spin wave excitation is illustrated in Fig. 3 with the help of micromagnetic simulations ${ }^{64}$ (see Ref. 62 for associated experimental results and specific simulation parameters). The sample consists of two cuboidal magnetic elements, aligned orthogonally and overlaid with a vertical separation of $10 \mathrm{~nm}$ (Fig. 3 (a)). Both the Fano transducer and waveguide are made of Permalloy but differ in thickness and width. When no bias magnetic field is applied, the shape anisotropy of the two elements compels the magnetisation to align along each element's long axis. The frequency spectrum of the sample is shown in Fig. 3 (b), from which the resonance frequencies of the transducer and waveguide are identified as $f_{\mathrm{t}}=11.5 \mathrm{GHz}$ and $f_{\mathrm{wg}}=9 \mathrm{GHz}$, respectively. A global microwave magnetic field of $11.5 \mathrm{GHz}$ frequency applied along the $x$-axis resonates with the transducer only. The precessing magnetisation of the transducer generates an oscillatory stray magnetic field that excites propagating spin waves in the waveguide (Fig. 3 (c)). 


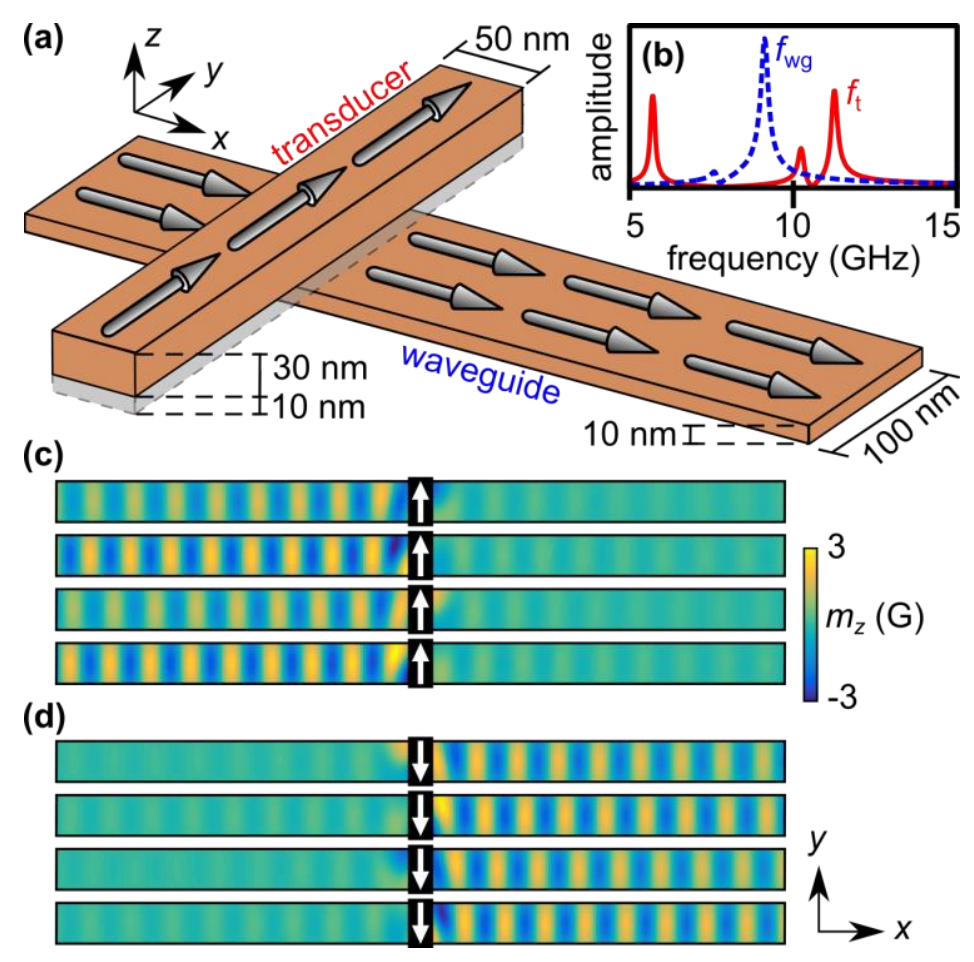

Fig. 3 (a) The geometry of the transducer-waveguide Fano resonance system. The arrows show the orientation of the static magnetisation within each element. (b) The frequency spectrum of the sample. (c)-(d) Snapshots of the out-of-plane component of the magnetisation of the waveguide are shown for time steps of $22 \mathrm{ps,}$, when the entire sample is excited at $11.5 \mathrm{GHz}$. The Fano transducer is magnetised (white arrows) parallel and antiparallel to the $y$-axis in panels (c) and (d), respectively. (After Ref. 46)

The Fano transducer has two roles in the design. Firstly, it localises the high frequency magnetic field to the nanoscale, enabling coupling to short wavelength spin waves. Secondly, it resonantly amplifies the incident field, ${ }^{65}$ helping achieve a stronger spin wave emission. In addition, the orientation of the transducer's magnetisation has a marked impact on its functionality. When the transducer is excited at resonance, its magnetisation precesses with a well-defined chirality. The handedness of this chirality leads to the unidirectional excitation of propagating spin waves, similar in manner to the water flow generated by a rotating water mill. In Fig. 3 (c), the magnetisation in the transducer is parallel to the $y$-axis, and spin waves are excited towards the negative $x$-direction. However, if the direction of the transducer's magnetisation is flipped (Fig. 3 (d)), the spin waves instead propagate towards the positive $x$-direction. Several periodically spaced Fano transducers create a periodic highfrequency magnetic field, ${ }^{65}$ leading to the idea of a resonant grating coupler discussed in detail in Refs. 63,66,67 (and also another Chapter of this Book). 
In a graded magnonic medium, one could consider the incident microwave magnetic field as tuned in frequency to some of its regions better than to others. One could therefore tune the microwave frequency so as to excite certain (specifically targeted) regions of the medium at resonance. The resonantly-driven magnetisation precession could then launch spin waves of finite wave vector into the adjacent regions, if such propagating spin wave modes are at all allowed by the dispersion relation in those regions. In this so-called Schlömann mechanism of spin wave excitation, ${ }^{55,56}$ the resonating regions of the sample do not confine spin waves and therefore are not characterised by a discrete spectrum. Hence, the mechanism is free from reliance on the resonance with discrete normal modes of well-defined parts of the sample, inherent to the case with Fano resonances. This means that the frequency tuning range of the emitted spin waves is no longer limited by the resonance linewidth of the Fano transducer. Instead, the tuning is determined by the graded magnonic index leading to a continuous distribution of the local FMR frequency, $f_{\mathrm{FMR}}(\boldsymbol{r})$, assigned to each and every point of the sample under the assumption of negligible impact of magnetisation gradients on the precession frequency. ${ }^{68}$ In other words, the local FMR frequency is defined by setting the wave vector to zero in the spin wave dispersion relation, with the latter defined by the local values of the magnetic parameters, field strength and static magnetisation orientation.

The mechanism is illustrated in Fig. 4. Specifically, we have studied a $10 \mu \mathrm{m}$ wide, $100 \mathrm{~nm}$ thick stripe of Permalloy, magnetised along its width. The magnetic charges dynamically induced at the stripe's left end by the magnetisation precession create a local increase in $f_{\mathrm{FMR}}(\boldsymbol{r})$, which then gradually decreases to the bulk value (i.e. the value far from the stripe ends) as the distance from the end increases (Fig. 4 (a)). The different values of $f_{\mathrm{FMR}}(\boldsymbol{r})$ correspond to different propagating spin wave modes, the dispersion of which is shown in Fig. 4 (b). Hence, when the stripe is excited by the uniform microwave magnetic field with a frequency matching $f_{\mathrm{FMR}}(\boldsymbol{r})$ at a particular distance from the end, the magnetisation at the distance gets resonantly excited and becomes a source of spin waves propagating into the stripe. The spin waves are imaged using the time-resolved scanning Kerr microscope (TRSKM), ${ }^{26,61}$ with the acquired images presented in Fig. 4 (c) and (d) for the excitation at frequencies of 5.76 and $7.52 \mathrm{GHz}$, respectively. Further measurements (not shown here) have demonstrated successful excitation of spin waves across a frequency range of more than $4 \mathrm{GHz}$, which far surpasses in bandwidth the Fano resonance assisted mechanism from Refs. 61,62. 

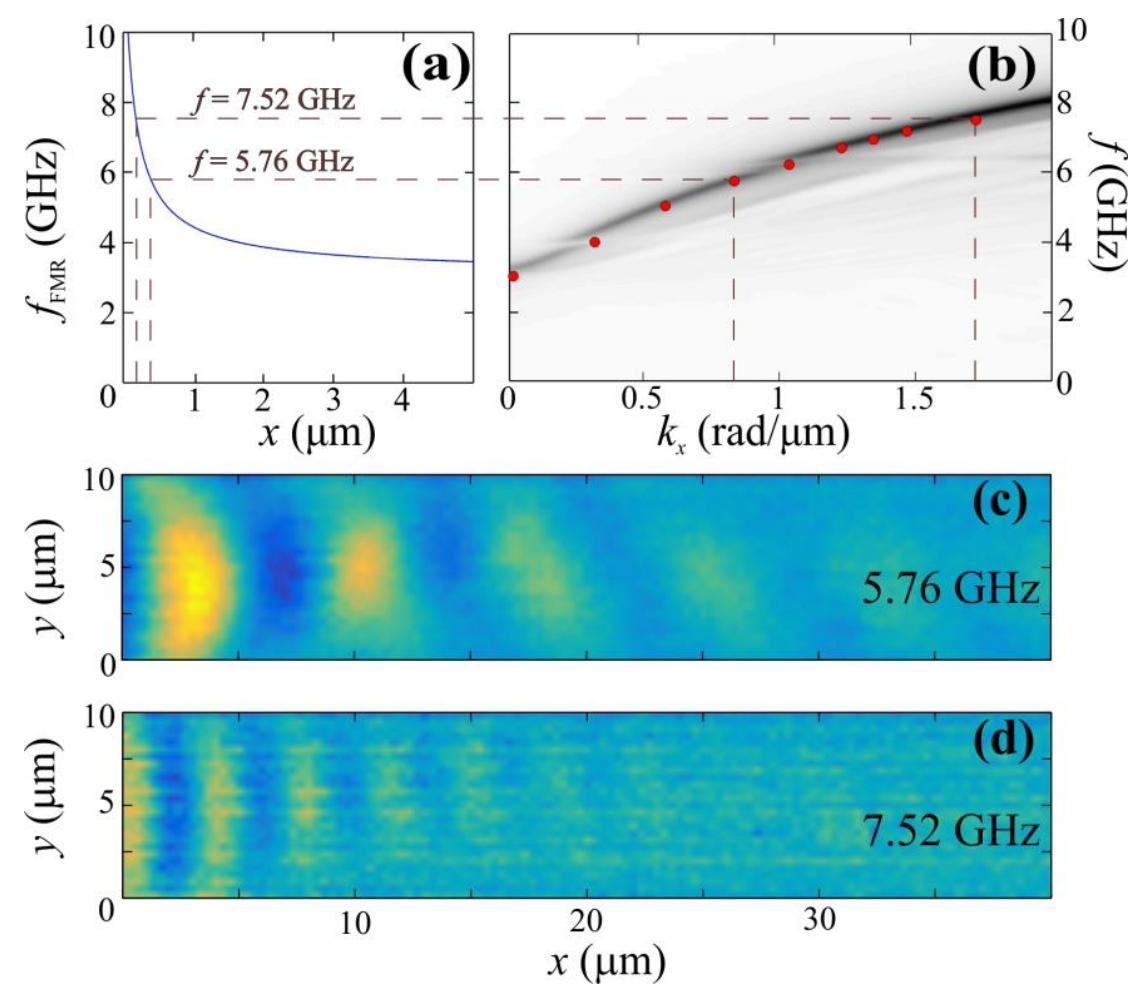

Fig. 4 (a) A cross-section of the calculated $f_{\mathrm{FMR}}(\mathbf{r})$ profile along the length of the stripe is shown for the first $5 \mu \mathrm{m}$ from its left end and $y=5 \mu \mathrm{m}$. (b) The spin wave dispersion calculated from simulations of an infinitely long stripe is shown by greyscale, with the overlaid red circles showing the points deduced from the measurements of the finite length stripe. For the dispersion calculation, the results of the simulations were spatially smoothed so as to mimic the experimental resolution. The horizontal dashed lines extending from panel (a) to (b) illustrate the correspondence between the source region and wave number of propagating spin waves excited at frequencies of 5.76 and $7.52 \mathrm{GHz}$, Kerr images of which are shown in panels (c) and (d) respectively.

A peculiar example of a graded magnonic landscape is given by magnetic domain walls, i.e. boundaries between regions of different magnetisation orientation. In particular, it has been recently shown that a domain wall can generate spin waves when excited by an external magnetic field or a spin-polarised current, ${ }^{69-74}$ while arrays of domain walls were proposed as spin wave grating couplers. ${ }^{75}$ The spin wave emission has traditionally been attributed to the effect of the domain wall oscillations. However, at least in two of the studies referenced above, the spin waves were observed to have the frequency of the driving stimuli, rather than twice its value (as one would expect for a nonlinear process of interaction between two oscillatory modes). So, to uncover the mechanism of the emission, we have developed a linear analytical theory in the exchange approximation using the formalism from Ref. 58. 

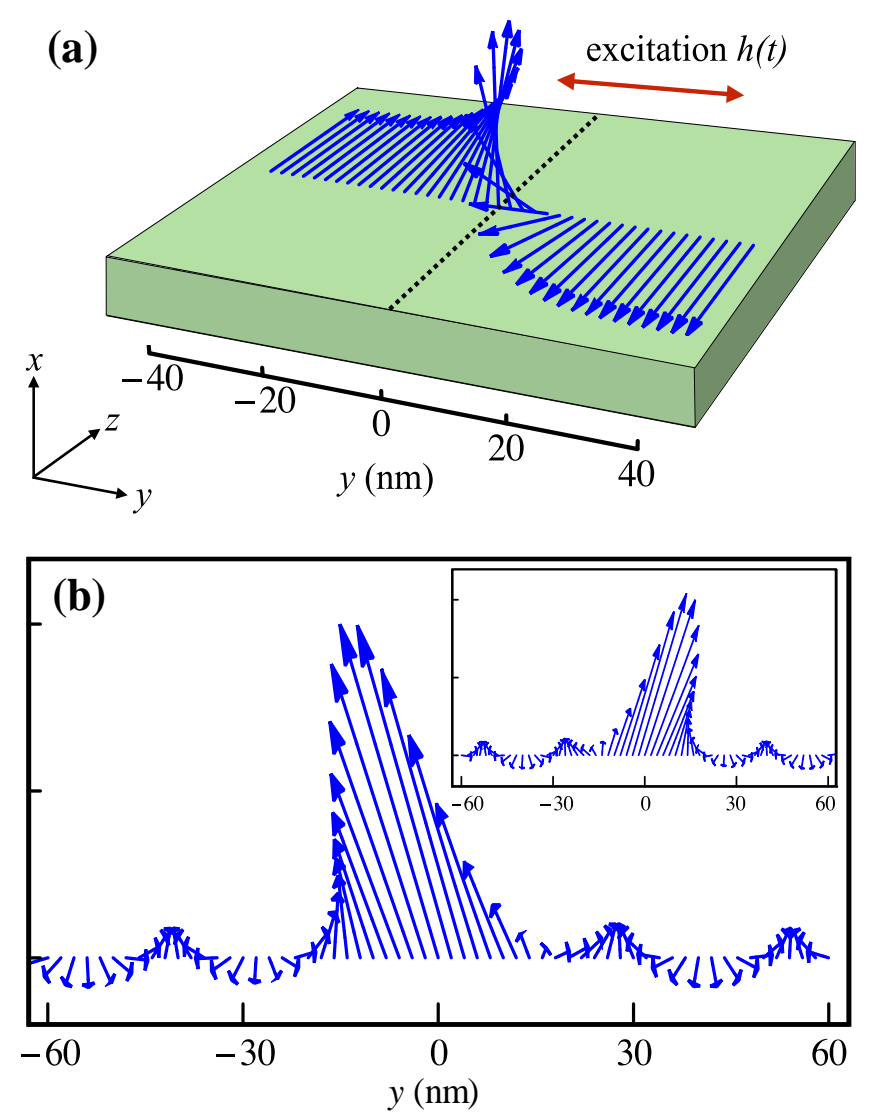

Fig. 5 (a) Geometry of a thin film extending infinitely in the $y-z$ plane with blue arrows showing the calculated magnetisation of a domain wall (centred at $y=0$ ) and adjoining antiparallel domains. The driving magnetic field is aligned along the $y$ direction. (b) Calculated magnetisation vectors at phase values of 0 (main plot) and $\pi$ (inset), showing spin waves emanating from the domain wall in (a).

Fig. 5 (a) shows a domain wall excited by a uniform microwave magnetic field oriented perpendicular to the magnetisation in both the domain wall and domains. Our calculations show that propagating spin waves at the frequency of the incident field are emitted from the domain wall (Fig. 5 (b)), as a result of a linear process. The underlying mechanism is similar to that proposed by Schlömann, ${ }^{55}$ except the domain wall creates a natural graded magnonic index landscape with a reduction of the local FMR frequency. This reduction prevents the incident microwave field from matching the local FMR frequency in any point of the sample. Although our theory is developed in the exchange approximation, we believe the described mechanism for spin wave generation is general and therefore applicable to the experimental observations referred to above. 
Curiously, we find that the graded magnonic index profile induced by a domain wall (that of the Pöschl-Teller potential well [76]) is naturally sized so that spin wave emission does occur and is locally optimised. For other values of the profile's aspect ratio, the emission could be suppressed or even completely eliminated, i.e. although a graded magnonic index may be able to generate spin waves, their emission is not guaranteed. As a final note of this section, we point out that the distinction between the spin wave emission mechanisms due to Fano resonances and graded magnonic index is very subtle. Indeed, depending on their taste, one could also consider the continuously varying local FMR frequency as an array of coupled Fano resonators, each with a slightly different yet discrete spectrum. At the same time, the spin wave emission from a domain wall could still be argued to be of resonant character, albeit with a large detuning from the domain wall resonance. A more detailed discussion of this will be presented elsewhere.

\section{Spin wave steering}

The functional medium element of the generic magnonic device shown in Fig. 1 (b) has two main purposes: to deliver the signals from the input to the output and, as magnonic devices can have multiple inputs and outputs, to steer the signals between them. A graded magnonic index between the inputs and outputs can be used to channel ${ }^{77-80}$ or focus ${ }^{81} /$ defocus ${ }^{82}$ spin waves, or to "cloak" an object from them, ${ }^{32}$ in analogy to a similar research topic in electromagnetics. ${ }^{25}$ Spin wave steering is the key prerequisite for creation of efficient magnonic interferometers, ${ }^{83-85}$ Boolean and analogue computing primitives, ${ }^{21,86-89}$ splitters (demultiplexers and inverse multiplexers) ${ }^{26,90-92}$ and combiners (multiplexers). ${ }^{93-95}$

Fig. 6 demonstrates the function of a magnonic inverse multiplexer formed by a Permalloy T-junction with $5 \mu \mathrm{m}$ wide features. ${ }^{26}$ The spin waves are excited in the central "leg" of the T-junction by a uniform microwave magnetic field through the Fano resonance assisted mechanism, as described in the previous section. The frequency of the incident microwave field is tuned to $f_{\mathrm{FMR}}$ of the leg, which acts as an element with a discrete spectrum. The sample was biased by a uniform in-plane static magnetic field, $\boldsymbol{H}_{\mathrm{B}}$, of $500 \mathrm{Oe}$ strength, applied either parallel to or slightly tilted from the long axis of the leg. Due to the magnetic shape anisotropy, the arms of the T-junction have a lower $f_{\mathrm{FMR}}$ compared to the leg. So, the resonantly excited magnetisation of the leg launches propagating magnetostatic spin waves into either one or both of the T-junction's arms, which act as elements with a continuous 
spectrum. The spin waves were imaged by the TRSKM and modelled using micromagnetic simulations.
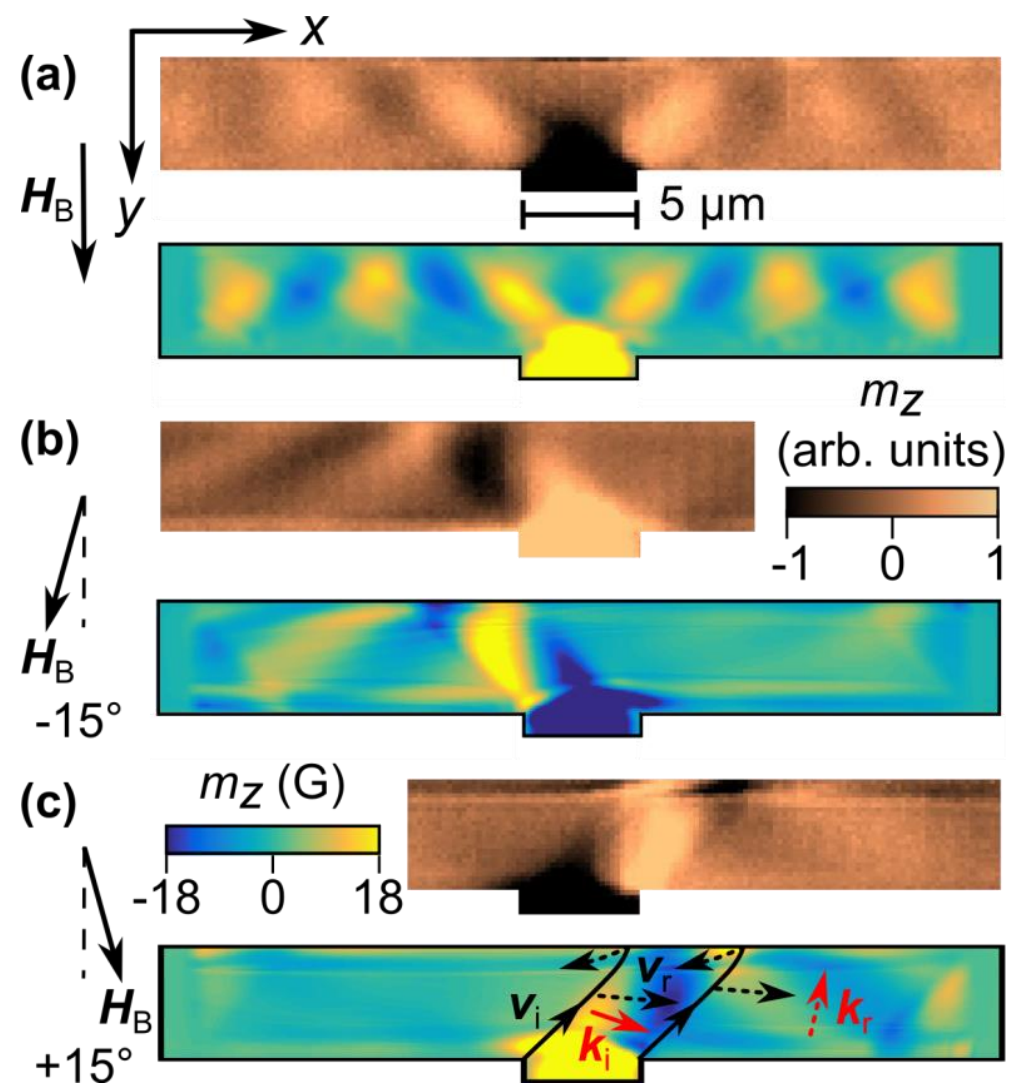

Fig. 6 Snapshots of spin waves propagating in the arms of the T-junction (after Ref. 26). The 500 Oe bias magnetic field $\boldsymbol{H}_{\mathrm{B}}$ is applied (a) parallel to and at angles of (b) $-15^{\circ}$ and (c) $+15^{\circ}$ relative to the leg of the junction. In each case, the top and bottom panels show results of the TRSKM imaging and micromagnetic simulations, respectively. The frequency of the continuous wave (cw) pump was $8.24 \mathrm{GHz}$ for experiments, while for simulations it was $7.62 \mathrm{GHz}$ in panel (a) and $7.52 \mathrm{GHz}$ in panels (b) and (c). In panel (c), the extracted directional vectors of the incident (index "i", solid lines) and reflected (index "r", dashed lines) group velocities $\boldsymbol{V}$ and wave vectors $\boldsymbol{k}$ are shown for $k_{x}=0.94 \mu \mathrm{m}^{-1}$. (After Ref. 26)

The observed switching of the spin wave propagation in the arms of the T-junction via the tilting of the in-plane bias magnetic field is a direct result of the graded magnonic index in the patterned structure and an excellent illustration of the opportunities in graded index magnonics in terms of spin wave steering. Firstly, Fig. 6 (a) shows that the phase fronts of the spin waves in the arms are somewhat tilted relative to their symmetry axis, even though 
the bias field is applied symmetrically. This is because the bias magnetic field does not fully saturate the magnetisation in the arms, which therefore tilts from their symmetry axes. At the same time, the energy flow along the arms dictates the direction of the group velocity, which is therefore bound to be parallel to their horizontal symmetry axis. Hence, the anisotropic dispersion of the magnetostatic spin waves (Fig. 2) leads to a small, non-zero angle between the directions of the group velocity and wave vector (phase velocity), explaining the tilt of the phase fronts. The same explains the tilts of the spin wave phase fronts observed in Fig. 4 (c) and (d). When the bias magnetic field is rotated from the symmetry axis by just $\pm 15^{\circ}$, we observe only one spin wave beam propagating into one of the arms (Fig. 6 (b) and (c)). The direction of the propagation is "switched" between the two arms by the sign of the tilt angle. In each case, the spin wave beam (emitted from the leg-arm junction) propagates at an oblique angle to the arm's axis, hits its edge, and is reflected into a much broader beam, propagating approximately along the arm's length. The incidence and reflection angles are different, again resulting from the anisotropy of the magnetostatic dispersion relation and the tilt of the static magnetisation.

We interpret our observations in terms of the graded magnonic index induced by the spatial variations of the orientations of the magnetisation and the value of the internal magnetic field in the sample. Using the convincing agreement between the measured and numerically simulated results, we apply the theory from Refs. 96,97 to the numerically computed static magnetisation and field distributions (Fig. 7 (a)) to derive the local directions of the wave vectors and group velocities of the propagating spin waves (shown Fig. 6 (c)). The confinement of the precessing magnetisation to the width of the T-junction's leg results in a broad $k_{x}$ spectrum (Fig. 7 (c)). For each $k_{x}$ value, the isofrequency curve corresponding to the frequency of the incident microwave field returns allowed (by the magnetostatic dispersion relation) values of $k_{y}$, while the normals to the isofrequency curves show the group velocity directions (Fig. 7 (d)-(h)). The field and magnetisation distributions in the arms are quite uniform along the $x$-axis starting from about $1 \mu \mathrm{m}$ from the leg-arm boundary, which ensures conservation of the $k_{x}$ value of the spin wave propagating across the arm's width. In contrast, the values of $k_{y}$ and the group velocity adjust adiabatically to the variation of the internal field magnitude and direction of the magnetisation. ${ }^{98}$ The non-uniformity also leads to a distributed partial reflection of the spin wave amplitude, with the group velocity directions of the reflected waves also shown in Fig. 7(c)-(h). The overall wave field is given by the superposition of the incident and scattered waves. 


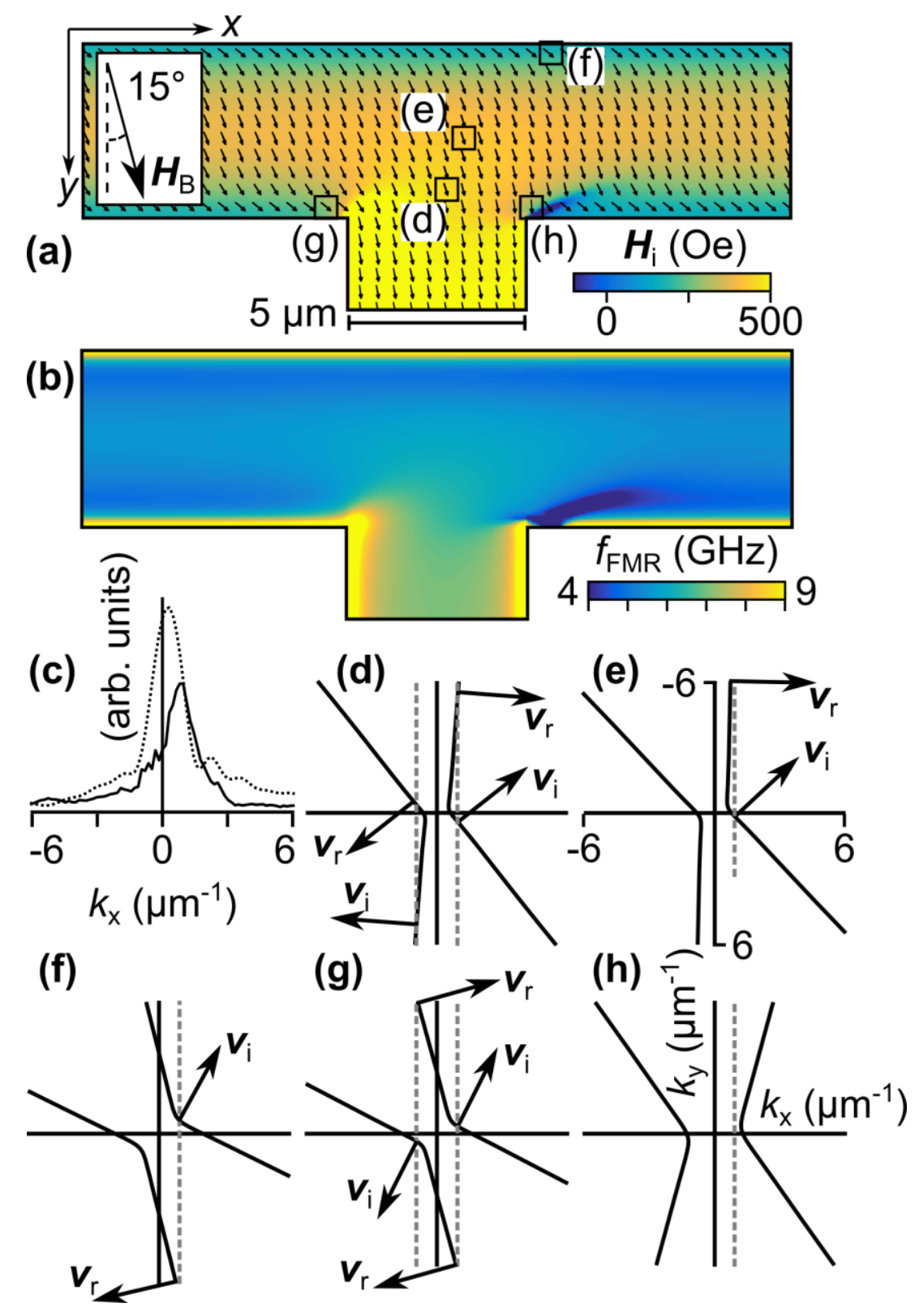

Fig. 7 (a) The calculated distributions of the static magnetisation (arrows) and the projection of the internal magnetic field onto the magnetisation (colour scale) are shown for the magnetic field of $\boldsymbol{H}_{\mathrm{B}}=500$ Oe applied at $15^{\circ}$ to the vertical symmetry axis. Each arrow represents the average of $5 \times 5$ mesh cells. (b) The calculated distribution of $f_{\mathrm{FMR}}(\boldsymbol{r})$ across the T-junction. (c) $k_{x}$ spectra of the dynamic magnetisation distributions across the leg and along the arms (amplified x5) of the Tjunction excited at $7.52 \mathrm{GHz}$ are shown by the dotted and solid curves, respectively. (d)-(h) Constructions of the isofrequency curves and the group velocities of the incident (index " $i "$ ) and reflected (index "r") beams are shown for the pixels boxed in (a), for the transverse wave vector components $k_{\mathrm{x}}= \pm 0.94 \mu \mathrm{m}^{-1}$ (as indicated by the dashed grey lines). (After Ref. 26) 
Apart from the small region at small wave vectors, the spin wave isofrequency curves depicted in Fig. 7 (d)-(h) consist of nearly straight lines. This leads to virtually the same direction of group velocity for a wide range of wave vectors, giving rise to the formation of spin wave caustic beams. ${ }^{47,96,97}$ This explains the strongly directional beam emitted from the leg-arm boundary for the tilted bias magnetic field, but not the absence of the other beam. Due to the inhomogeneities of the internal field and magnetisation (Fig. 7 (a)), the beam curves slightly and experiences distributed scattering, with the group velocities of the scattered waves being roughly aligned with the arm's length (Fig. 7(d),(e)). The group velocity of the reflected beam switches direction near the far edge of the arm (Fig. 7 (f)), leading to the phenomenon of "back reflection". ${ }^{96}$ The reflected beam is confined by the nonuniform demagnetising field and magnetisation near the arm's edge. In addition to this, some spin waves with small (negative or positive) $k_{x}$ values are not supported in parts of the magnetic landscape at all. In Fig. 7 (h), for example, there is no intersection between the line $k_{x}=0.94 \mu \mathrm{m}^{-1}$ and the isofrequency curves, giving rise to a "forbidden" path for spin waves of certain wave vector. Finally, and quite surprisingly, we find that the beam formed from spin waves with negative $k_{x}$ values cannot possibly propagate into the left arm of the junction (for the bias field direction in Fig. 7). Indeed, the beam is curved into the nearest edge of the left arm from which it is then scattered backwards into the right arm (Fig. 7 (g)). The observed complete disappearance of one of the two beams in favour of the other one would be impossible without the graded distribution of the magnonic index. The anisotropic dispersion is also required for the effects to take place, but on its own it could only lead to a tilt of both beams and asymmetry of their intensities. ${ }^{92}$

Fig. 6 clearly shows that the spin wave beam initiated near the leg-arm boundary then propagates along the arms of the structure as prescribed by the direction of the bias magnetic field. However, due to the same widths of the leg and arms of the structure, the beams are also quite wide and not as distinct as one could wish. So, Fig. 8 presents results of simulations for a Permalloy $\mathrm{T}$-junction that has a narrower ( $1 \mu \mathrm{m}$ wide) leg, which leads to a better-defined spin wave caustic beam propagating into one arm of the structure. The smaller width of the leg leads to the higher frequency of its quasi-uniform mode and to the observed smaller cross-section of the spin wave beam excited at the frequency into the right arm of the structure. Notably, the observations and interpretations developed for the wider leg sample also remain valid here. 

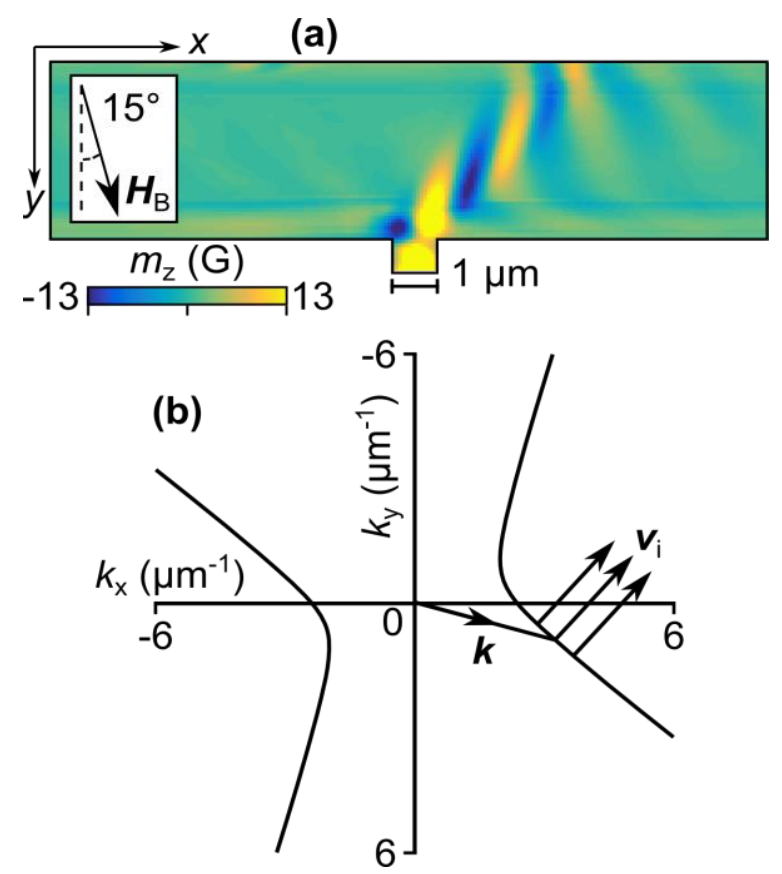

Fig. 8 (a) A numerically calculated snapshot of the dynamic out-of-plane component of the magnetisation in the T-junction. The bias magnetic field $\boldsymbol{H}_{\mathrm{B}}=500 \mathrm{Oe}$ is applied at an angle of $\alpha=15^{\circ}$ relative to the leg of the T-junction driven by a microwave magnetic field of $10.3 \mathrm{GHz}$ frequency. (b) An example isofrequency curve calculated for the frequency of excitation and with the internal field and magnetisation corresponding to the region at the centre of the arms (above the leg). The arrows represent examples of the wave vector $(\boldsymbol{k})$ and group velocity $\left(\boldsymbol{v}_{\mathrm{i}}\right)$ vectors: the mutual collinearity of the group velocity vectors gives rise to the spinwave caustic beam observed in panel (a). (After Ref. 26)

The non-uniformity of the magnetisation and internal magnetic field plays the key role in defining the spatial variation of the graded magnonic index and thereby in steering the direction of the spin wave propagation in the T-junction. However, the other key ingredient the anisotropy of the magnetostatic spin wave dispersion - is only (strongly) present at micrometre to millimetre length scales, impeding miniaturisation of any magnonic devices that would exploit the type of spin wave steering discussed here. In contrast, the nonuniformity of the internal magnetic field and the magnetisation persists to much shorter length scales and could still lead to useful device concepts. Moreover, on the nanometre length scales, the non-uniform exchange field (completely neglected here) becomes more important and could therefore be exploited, while additional opportunities arise from the use of the highly localised magnetic field due to magnetic domain walls. ${ }^{70-75,83}$ In this context, the main challenge is that the configurations of the internal magnetic field and the static 
magnetisation in magnetic nano- and micro-structures are not arbitrary but are determined by the magnetostatic Maxwell equations. This limits the range of magnetic configurations that could be exploited and favours alternative pathways to creation of the graded magnonic index.

\section{Spin wave output}

In research labs, the spin wave outputs from magnonic devices are often detected using Kerr microscopy ${ }^{17,26,33,61-63,72}$ and Brillouin light scattering, ${ }^{9,16,22,59,77,91-93}$ (the two most popular magneto-optical techniques in magnonics). However, the outputs of realistic magnonic devices will need to be more congenial to logic circuitry of interest, with their nature depending on the envisaged purpose of the magnonic chip. For example, spin waves can be interfaced with high frequency electrical signals inductively ${ }^{19}$ or magnetoresistively, ${ }^{13,14}$ or their action could be encoded into the micromagnetic configuration of domain walls ${ }^{99}$ or magnetic nanoelements, ${ }^{9,16,17}$ or both. ${ }^{100}$ For example, propagating spin waves have been demonstrated to be able to drive domain walls across magnetic samples, ${ }^{101}$ which could be used either to toggle binary logic states or for a memristor-type function. ${ }^{14}$

Arguably, the most important missing element in the technological toolbox of modern magnonics is the direct interfacing of two or more magnonic devices in series. The only demonstration of this kind was done using micromagnetic simulations in Ref. 86, with none reported experimentally. It is more challenging to realise the fan-out function, whereby the output of one device (e.g. a logic gate) is connected and can drive the input of more than one identical devices. However, the expanding use of ultrathin films of yttrium-iron garnet (YIG), ${ }^{67}$ which have naturally low magnetic damping, ${ }^{102}$ and the recent demonstration of low damping in metallic CoFe alloys ${ }^{103,104}$ should help further experimental progress in this direction as well as in magnonics, in general.

In the context of detection of output signals from magnonic devices, the graded magnonic index could be used to fine-tune the wavelengths of propagating spin waves to the range of values optimal for a specific output detection method. Indeed, in a reverse process to the Schlömann excitation mechanism, an increase of the local FMR frequency $f_{\mathrm{FMR}}(\mathbf{r})$ in a medium with a positive dispersion results in an increase of the spin wave wavelength..$^{98,105}$ Thus, a scheme can be realised in which the data or signal processing is done by spin waves 
of shorter wavelength that are then upscaled to a larger wavelength, e.g. about twice the size of the detector, thereby optimising the spin wave coupling to the external circuitry.

In the same spirit, we have shown in Ref. 106 that the transducer-waveguide Fano resonance system shown in Fig. 3 can be used in the reverse direction: to couple an incident propagating spin wave to the uniform precession in the "transducer", with the latter acting in this case as a receiving antenna. As a result, the spin wave can be fully absorbed, leading to a pronounced uniform precession of the receiving element. This uniform precession could then be more easily outcoupled to the external circuitry, in a function that is similar to that of the case of the graded magnonic index discussed in the previous paragraph. The only experimental demonstration of this kind was reported in Ref. 67, albeit without a detailed discussion of the detection mechanism.

\section{Spin wave control and magnonic devices}

The magnonic dispersion itself and the spin wave excitation, steering and detection mechanisms discussed in the preceding sections of this Chapter are all determined by the internal magnetic field and magnetisation texture in the sample. Hence, the mechanisms lend themselves readily to external control, which could be realised through application of the bias magnetic field or even the history of its application. The former can enable construction of magnetically tuneable magnonic devices, while the latter property describes devices reprogrammable by the applied (and eventually removed) magnetic field. However, the physics of magnetic switching and control of micromagnetic textures (either by a magnetic field or otherwise, e.g. electrically, optically, acoustically, spintronically etc) forms a separate and extremely broad research field, which is beyond the scope of this Chapter. So, in this section, we discuss only a few examples of magnonic devices that exploit the graded magnonic index and / or Fano resonances and can be controlled either by the applied magnetic field or by switching their micromagnetic configuration.

The results of our TRSKM imaging experiments and micromagnetic simulations presented in Fig. 6 demonstrate the efficiency by which an external bias magnetic field can be used to steer magnetostatic spin waves across a T-junction. ${ }^{26}$ When the bias magnetic field is applied symmetrically along the leg, the T-junction acts as a magnonic inverse multiplexer, i.e. a spin wave splitter. ${ }^{91}$ The demonstrated magnetic field control of the spin wave beam 
enables the T-junction to be also used as an analogue time division demultiplexer, i.e. a device that can steer time-separated signals between different outputs. The switching of the 500 Oe magnetic field from $+15^{\circ}$ to $-15^{\circ}$ is equivalent to applying a constant bias field of 486 Oe along the leg and toggling a control magnetic field of \pm 117 Oe to switch the signal between outputs. A similar device fabricated from YIG could be switched by a control field of \pm 127 Oe for the constant bias magnetic field of 1153 Oe. ${ }^{92}$

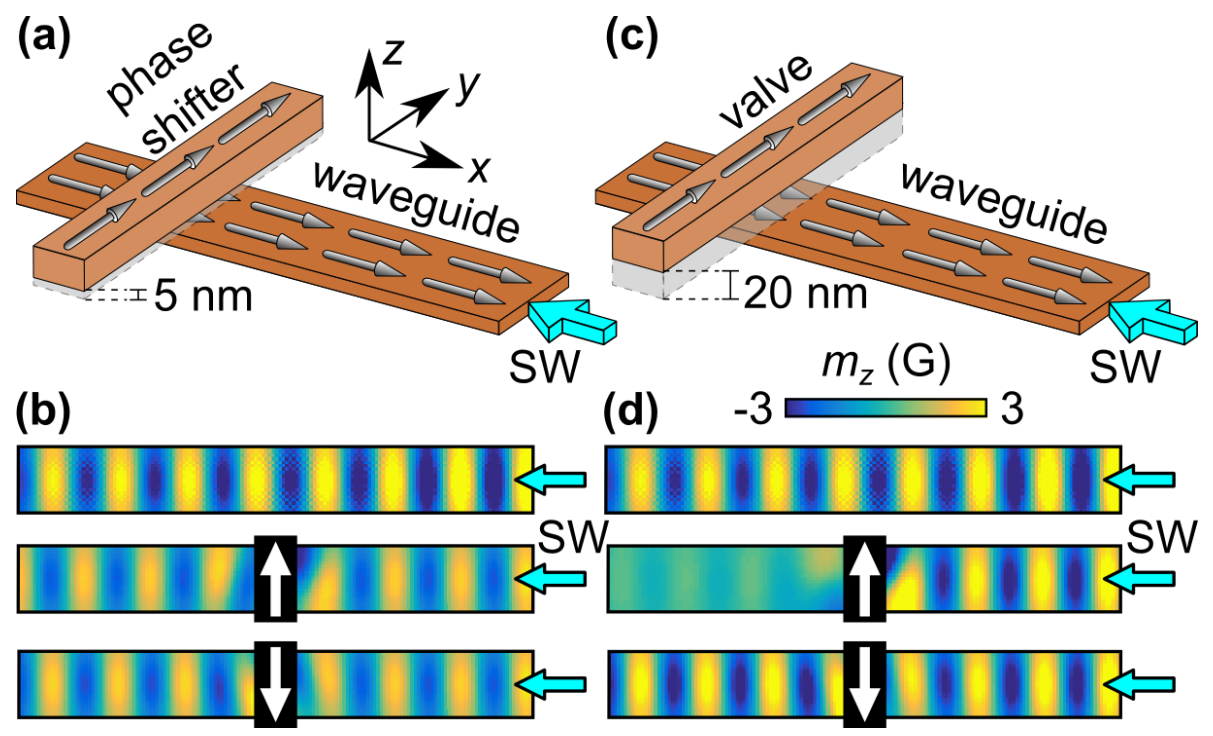

Fig. 9 (a) The Fano control element is positioned $5 \mathrm{~nm}$ above the waveguide. A spin wave (SW) excited elsewhere propagates along the waveguide in the negative $x$ direction. (b) The first (top) snapshot shows the out-of-plane component of magnetisation $\left(m_{z}\right)$ when the control element is absent. The second (middle) and third (bottom) snapshots show $m_{z}$ with the control element present and magnetized parallel and antiparallel to the $y$-axis, respectively. (c)-(d) The same as (a)-(b) but for the control element positioned $20 \mathrm{~nm}$ above the waveguide. (After Ref. 46)

Magnetically reprogrammable magnonic devices can be built using Fano resonances, the function of which could be "programmed" by switching the magnetisation of the Fano resonator (above a magnonic waveguide) discussed earlier in the context of spin-wave excitation and detection, i.e. the magnonic transducer from Fig. 3. In particular, this Fano resonator can act as a control element performing the function of either a valve or phase shifter, depending on the distance between the element and waveguide (Fig. 9). ${ }^{106}$ For one orientation of the element's magnetisation (the bottom images in panels (b) and (d)), the 
element does not couple to the propagating spin wave at all. However, for the opposite orientation of the magnetisation (the middle images in panels (b) and (d)), the stray magnetic field generated by a spin wave passing underneath can resonantly excite its precession. This precession is then partly re-emitted (in the same manner as the process shown in Fig. 3) as a spin wave propagating in the same direction as the originally incident wave, but with opposite phase. For the $20 \mathrm{~nm}$ element-to-waveguide separation, the partly transmitted original and the re-emitted spin waves have similar amplitudes and opposite phases. Thus, they nearly cancel each other, yielding the observed valve functionality. For the $5 \mathrm{~nm}$ element-to-waveguide separation, the incident spin wave is first fully converted into the element's precession and then fully re-emitted with a $180^{\circ}$ phase shift. In this case, no interference occurs, since there is no directly transmitted wave, and only the observed phase shifter functionality is observed.

The described methods of spin-wave excitation, control and detection can be used to construct a complete magnonic logic architecture. The simplest of logic gates is the NOT gate, and this can be straightforwardly realised using the scheme discussed in Fig. 9 (d). The binary input, which can take the values 0 or 1 , is assigned to the polarity of the valve's static magnetisation to be respectively towards negative and positive $y$. A spin wave detector, positioned on the waveguide past the valve, records an output of 1 if non-zero (i.e. above a set threshold) spin-wave amplitude is detected, or 0 if spin waves are absent. Hence, the valve can act as a simplistic magnonic NOT gate, as an input of 0 generates an output of 1 , and vice versa. Of course, this will require a subsequent amplification stage if the output is to be used in another magnonic logic gate.

Gates more sophisticated than a NOT gate require two input signals. This can be implemented by considering either two control elements, or two input transducers with a shared waveguide. For example, a XNOR gate can be constructed using two spin wave phase shifters combined to form a magnonic interferometer shown in Fig. 10 (a). Here, the two phase shifter elements are positioned $5 \mathrm{~nm}$ above each branch of the interferometer. The magnetisation follows the twists of the interferometer, the symmetry of which causes the propagating spin wave to split equally between its two branches. If the magnetisations in both phase shifters are parallel, i.e. input $(0,0)$ or $(1,1)$, the spin waves from the branches will interfere constructively upon recombination. Hence, a spin wave will be observed by the detector, i.e. output of 1 . If instead the phase shifters are antiparallel in the magnetisation 
polarity, i.e. input $(0,1)$ or $(1,0)$, the phase of one spin wave is shifted by $180^{\circ}$. So, the spin waves interfere upon recombination destructively, generating an output of 0 . This action is shown in Fig. 10 (b), where the results of micromagnetic calculations are presented for the $(0,0)$ and $(0,1)$ inputs.
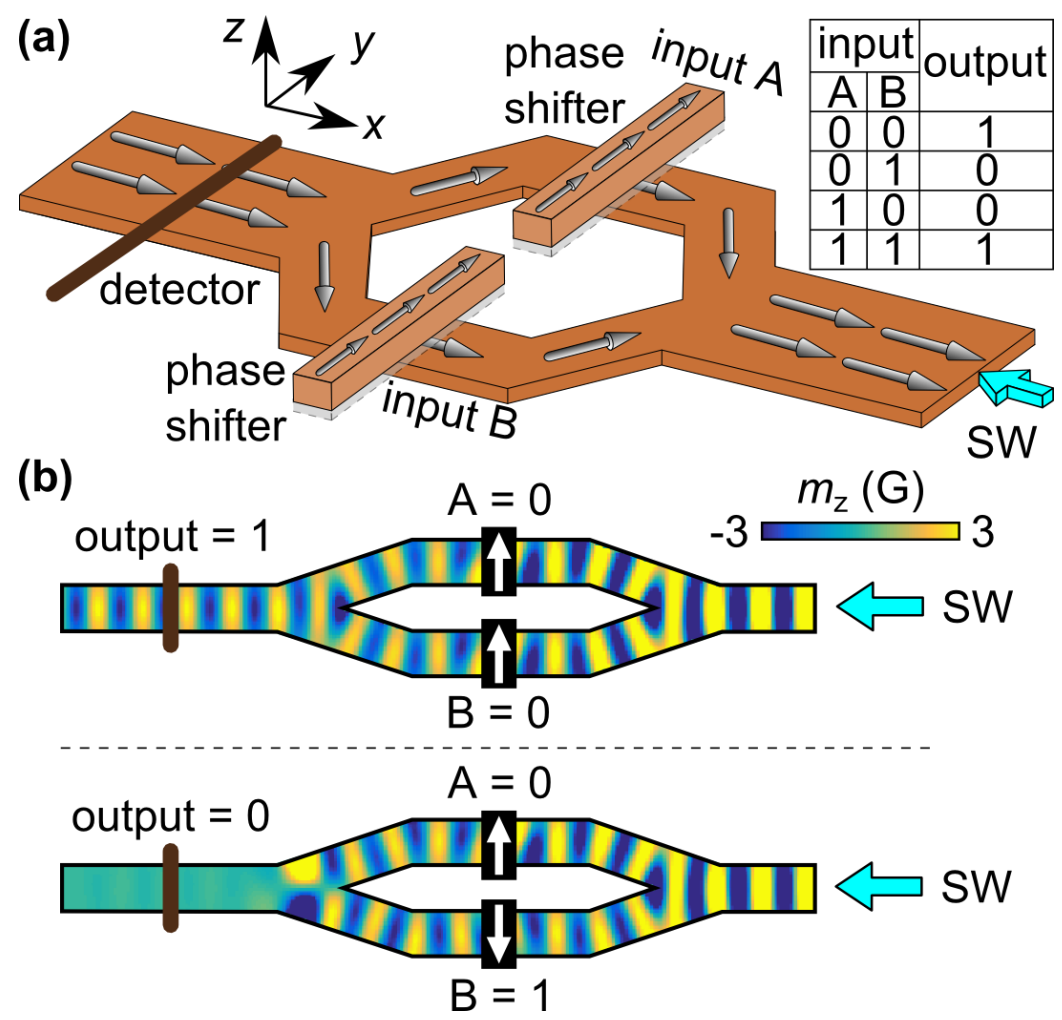

Fig. 10 (a) Magnonic XNOR gate realised as a spin-wave interferometer. The directions of the static magnetisation and spin wave (SW) propagation in the interferometer are shown. The magnonic phase-shifter elements are positioned above each branch of the interferometer. (b) Snapshots of the out-of-plane component of magnetisation $\left(m_{\mathrm{z}}\right)$ are shown for two of the four possible XNOR gate input combinations, as calculated using micromagnetic simulations. (After Ref. 46)

Fig. 11 (a) shows a magnonic NAND gate constructed using two input transducers. Similar to the encoding used in Fig. 10 (a), the transducers' static magnetisation polarity, at A and $\mathrm{B}$, is encoded by 0 when the magnetisation is aligned along the positive and negative $y$ directions, respectively, and encoded by 1 for the reversed case. The entire sample is excited globally by a harmonic microwave field. As shown in the calculated snapshots of spin wave propagation in Fig. 11 (b), spin waves are only absent from the centre of the waveguide when 
an input of $(1,1)$ is used. The NAND gate functionality is therefore obtained, as demonstrated in the truth table shown in the inset of Fig. 11 (a).

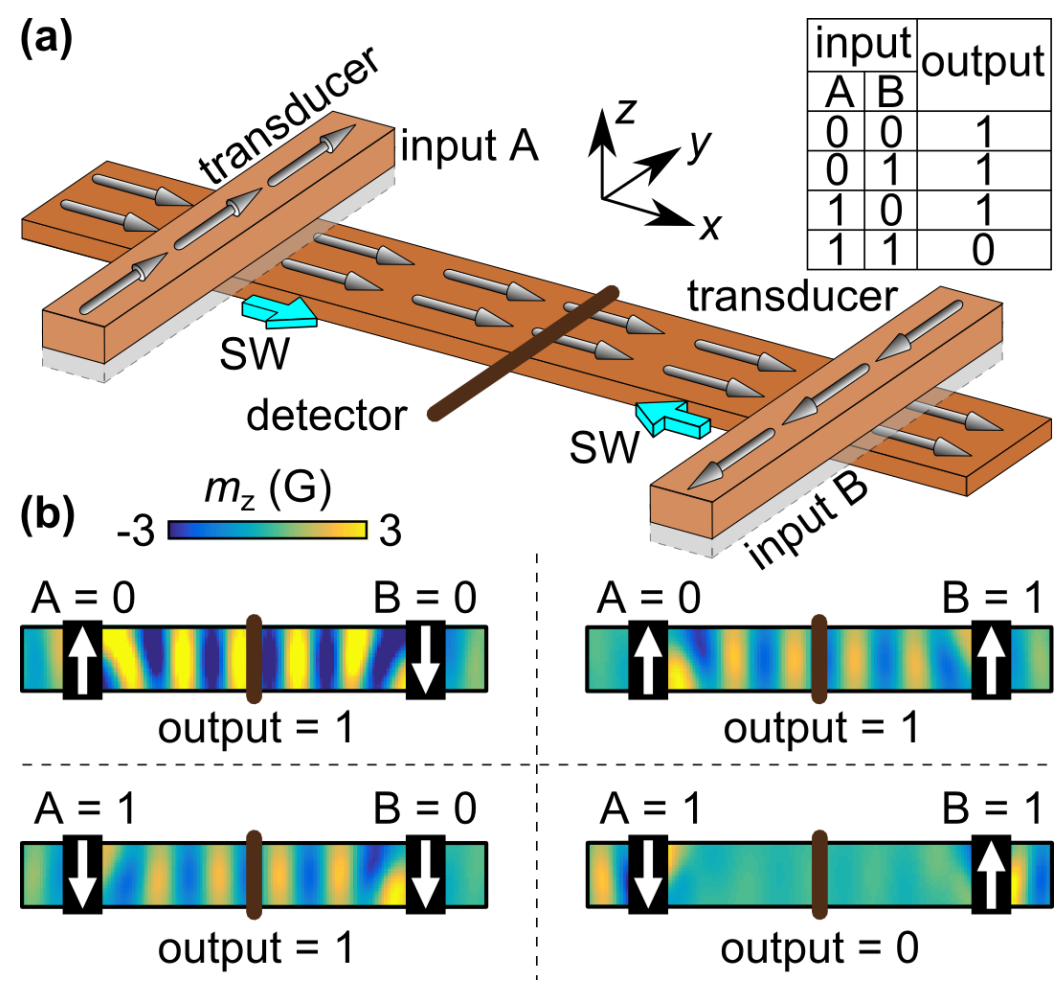

Fig. 11 (a) Magnonic NAND gate realised as a pair of two transducers positioned on a shared waveguide. (b) Snapshots of $m_{z}$ corresponding to the same moment of time are shown for the four possible NAND gate input combinations. (After Ref. 46)

\section{Conclusions and outlook}

As conventional electronics is beginning to become restricted in its potential for growth, the research and development of technologies that use alternative means of data processing and communication is gathering significant attention. Among others, magnonic technology promises devices that will have a low footprint, moderately high operational frequencies and intrinsic non-volatility, with scope for efficient interfacing with electronic and other emerging research devices. However, in order to advance the development of spin wave devices, research should not just focus on the design, experimental construction and miniaturisation of prototype magnonic devices within existing paradigms. While deepening our understanding of known, fundamental spin wave concepts, we should also proactively 
search for novel spin wave phenomena and use this knowledge to imagine new devices, which are designed to harness the unique properties of spin waves.

In this Chapter, we have attempted to demonstrate the interesting physics and highlight technical opportunities associated with the graded magnonic index and spin wave Fano resonances. The research into these two exciting (yet underexplored in magnonics) wave phenomena is rapidly gaining momentum, as evidenced by the increased attention they receive throughout this book. We foresee three main research avenues for further advances and the ultimately bright future of these fields. First, the science of spin wave propagation in media with a graded magnonic index and / or Fano resonators is unchartered territory in terms of mathematical physics, with lots of challenges and surprises awaiting theoreticians' attention. Second, similarly vast opportunities will open to experts in numerical micromagnetic simulations that will venture into the world of media and devices including compositionally modulated structural elements that extend into three dimensions. And last but not least, the continuing progress in nanotechnology and materials science and the most recent advances in reducing the magnetic damping hold promise for the latter exciting phenomena to be observed experimentally and implemented within realistic magnonic device architectures.

\section{Acknowledgements}

The research leading to these results has received funding from the Engineering and Physical Sciences Research Council of the United Kingdom (Project Nos. EP/L019876/1, EP/L020696 and EP/P505526/1), and from the European Union's Horizon 2020 research and innovation program under Marie Skłodowska-Curie Grant Agreement No. 644348 (MagIC).

1 V. V. Kruglyak, S. O. Demokritov, and D. Grundler, "Magnonics”, J. Phys. D - Appl. Phys. 43, 264001 (2010).

2 S. A. Nikitov, D. V. Kalyabin, I. V. Lisenkov, A. N. Slavin, Y. N. Barabanenkov, S. A. Osokin, A. V. Sadovnikov, E. N. Beginin, M. A. Morozova, Y. P. Sharaevsky, Y. A. Filimonov, Y. V. Khivintsev, S. L. Vysotsky, V. K. Sakharov, and E. S. Pavlov, "Magnonics: a new research area in spintronics and spin wave electronics", Physics-Uspekhi 58, 1002 (2015). 
3 A. I. Akhiezer, V. G. Bar'yakhtar, and S. V. Peletminskii, "Spin waves" (NorthHolland, Amsterdam, 1968).

4 A. G. Gurevich and G. A. Melkov, "Magnetization Oscillations and Waves" (Chemical Rubber Corp., New York, 1996).

5 H. Al-Wahsh, A. Akjouj, B. Djafari-Rouhani, and L. Dobrzynski, "Magnonic circuits and crystals", Surf. Sci. Rep. 66, 29 (2011).

6 L. J. Heyderman and R. L. Stamps, "Artificial ferroic systems: novel functionality from structure, interactions and dynamics”, J. Phys. Condens. Matter 25, 363201 (2013).

$7 \quad$ M. Krawczyk and D. Grundler, "Review and prospects of magnonic crystals and devices with reprogrammable band structure”, J. Phys. Condens. Matter 26, 123202 (2014).

$8 \quad$ J. Rychly, P. Gruszecki, M. Mruczkiewicz, J. W. Klos, S. Mamica, and M. Krawczyk, "Magnonic crystals - Prospective structures for shaping spin waves in nanoscale", Low Temp. Phys. 41, 741 (2015).

9 G. Gubbiotti, S. Tacchi, M. Madami, G. Carlotti, A. O. Adeyeye, and M. Kostylev, "Brillouin light scattering studies of planar metallic magnonic crystals", J. Phys. D - Appl. Phys. 43, 264003 (2010).

10 B. Lenk, F. Garbs, H. Ulrichs, N. Abeling, and M. Munzenberg, "Photo-magnonics", Top. Appl. Phys. 125, 71 (2013).

11 A. M. Kalashnikova, A. V. Kimel, and R. V. Pisarev, "Ultrafast opto-magnetism", Physics-Uspekhi 58, 969 (2015).

12 G. E. W. Bauer, E. Saitoh, and B. J. van Wees, "Spin caloritronics", Nature Mater. 11, 391 (2012).

$13 \mathrm{~S}$. Bonetti and J. Akerman, "Nano-contact spin-torque oscillators as magnonic building blocks", Top. Appl. Phys. 125, 177 (2013).

14 N. Locatelli, V. Cros, and J. Grollier, "Spin-torque building blocks", Nature Mater. 13, 11 (2014).

15 A. V. Chumak, V. I. Vasyuchka, A. A. Serga, and B. Hillebrands, "Magnon spintronics", Nature Phys. 11, 453 (2015).

16 A. O. Adeyeye and S. Jain, "Coupled periodic magnetic nanostructures (invited)", J. Appl. Phys. 109, 07 B903 (2011).

17 A. Barman and A. Haldar, "Time-domain study of magnetization dynamics in magnetic thin films and micro- and nanostructures", Solid St. Phys. 65, 1 (2014).

18 V. E. Demidov and S. O. Demokritov, "Magnonic waveguides studied by microfocus Brillouin light scattering”, IEEE Trans Magn. 51, 0800215 (2015).

19 D. Grundler, “Nanomagnonics”, J. Phys. D - Appl. Phys. 49, 391002 (2016).

20 A. A. Serga, A. V. Chumak, and B. Hillebrands, "YIG Magnonics", J. Phys. D - Appl. Phys. 43, 264002 (2010).

21 A. Khitun, M. Q. Bao, and K. L. Wang, "Magnonic logic circuits", J. Phys. D - Appl. Phys. 43, 264005 (2010). 
22 A. V. Chumak, A. D. Karenowska, A. A. Serga, and B. Hillebrands, "The dynamic magnonic crystal: New horizons in artificial crystal based signal processing", Top. Appl. Phys. 125, 243 (2013).

23 L.D. Landau and E.M. Lifshitz, "Quantum Mechanics: Non-Relativistic Theory" (Oxford, Pergamon Press, 1977).

24 E. W. Marchand, "Gradient Index Optics" (London, Academic Press, 1978).

25 U. Leonhardt and T. G. Philbin, "Geometry and light: The science of invisibility" (Mineola, New York, Dover Publications, Inc, 2010).

26 C. S. Davies, A. Francis, A. V. Sadovnikov, S. V. Chertopalov, M. T. Bryan, S. V. Grishin, D. A. Allwood, Y. P. Sharaevskii, S. A. Nikitov, and V. V. Kruglyak, "Towards graded-index magnonics: Steering spin waves in magnonic networks", Phys. Rev. B 92, 020408(R) (2015).

27 C. S. Davies and V. V. Kruglyak, "Graded-index magnonics", Low Temp. Phys. 41, 976 (2015).

28 Y. I. Gorobets and S. A. Reshetnyak, "Reflection and refraction of spin waves in uniaxial magnets in the geometrical-optics approximation", Techn. Phys. 43, 188 (1998).

29 S. A. Reshetnyak, "The approximation of geometrical optics for bulk spin waves in spatially inhomogeneous ferromagnetic insulators with an exchange defect", Low Temp. Phys. 30, 398 (2004).

30 D.-E. Jeong, D.-S. Han, S. Choi, S.-K. Kim, "Refractive index and Snell's law for dipole-exchange spin-waves in a confined planar structure", SPIN 1, 27 (2011).

31 P. Gruszecki, J. Romero-Vivas, Y. S. Dadoenkova, N. N. Dadoenkova, I. L. Lyubchanskii, and M. Krawczyk, "Goos-Hänchen effect and bending of spin wave beams in thin magnetic films", Appl. Phys. Lett. 105, 242406 (2014).

32 M. Elyasi, C. S. Bhatia, C. W. Qiu, and H. Yang, "Cloaking the magnons", Phys. Rev. B 93, 104418 (2016).

33 J. Stigloher, M. Decker, H. S. Korner, K. Tanabe, T. Moriyama, T. Taniguchi, H. Hata, M. Madami, G. Gubbiotti, K. Kobayashi, T. Ono, and C. H. Back, "Snell's law for spin waves", Phys. Rev. Lett. 117, 037204 (2016).

34 X. J. Xing and Y. Zhou, "Fiber optics for spin waves", NPG Asia Mater. 8, e246 (2016).

35 W. C. Yu, J. Lan, R. Q. Wu, and J. Xiao, "Magnetic Snell's law and spin-wave fiber with Dzyaloshinskii-Moriya interaction”, Phys. Rev. B 94, 140410 (2016).

36 S. O. Demokritov (Editor), "Spin wave confinement" (Pan Stanford, 2008).

37 U. Fano, "Effects of configuration interaction on intensities and phase shifts", Phys. Rev. 124, 1866 (1961).

38 A. E. Miroshnichenko, S. Flach, and Y. S. Kivshar, "Fano resonances in nanoscale structures", Rev. Mod. Phys. 82, 2257 (2010).

39 H. Al-Wahsh, E. H. El Boudouti, B. Djafari-Rouhani, A. Akjouj, T. Mrabti, and L. Dobrzynski, "Evidence of Fano-like resonances in mono-mode magnetic circuits", Phys. Rev. B 78, 075401 (2008). 
40 H. Al-Wahsh, "Evidence of Fano-like resonances in mono-mode magnetic circuits", Eur. Phys. J. B 73, 527 (2010).

41 G. E. Moore, "Cramming more components onto integrated circuits", Electronics 38, 8 (1965).

42 International Technology Roadmap for Semiconductors (ITRS) 2013 edition: Emerging Research Devices. http://www.itrs.net/Links/2013ITRS/Summary2013.htm (accessed 10 February 2015).

43 M. Mitchell Waldrop, "The chips are down for Moore's law", Nature 530, 144 (2016).

44 "Electronics, Encyclopedia Britannica". Encyclopedia Britannica. September 2016. https://www.britannica.com/technology/electronics (accessed 10 January 2017).

$45 \quad$ R. Lomas, "The Man Who Invented the Twentieth Century: Nikola Tesla, Forgotten Genius of Electricity" (Headline Publishing Group, London, 1999).

46 C. S. Davies, Y. Au, and V. V. Kruglyak, "Prototype magnonic device development", p. 54 in "Magnetics Technology International" (UKIP Media, Surrey, 2015); http://www.ukipme.com/pub-magnetics.php (accessed 18 January 2017).

47 V. Veerakumar and R. E. Camley, "Focusing of spin waves in YIG thin films", IEEE Trans. Magn. 42, 3318 (2006).

48 I. Dzyaloshinsky, "A thermodynamic theory of weak ferromagnetism of antiferromagnetics", J. Phys. Chem. Solids 4, 241 (1958).

49 T. Moriya, "Anisotropic superexchange interaction and weak ferromagnetism", Phys. Rev. 120, 91 (1960).

50 V. K. Dugaev, P. Bruno, B. Canals, and C. Lacroix, "Berry phase of magnons in textured ferromagnets", Phys. Rev. B 72, 024456 (2005).

51 V. S. Tkachenko, A. N. Kuchko, and V. V. Kruglyak, "An effect of the curvature induced anisotropy on the spectrum of spin waves in a curved magnetic nanowire", Low Temp. Phys. 39, 163 (2013).

52 R. Streubel, P. Fischer, F. Kronast, V. P. Kravchuk, D. D. Sheka, Y. Gaididei, O. G. Schmidt, and D. Makarov, "Magnetism in curved geometries", J. Phys. D - Appl. Phys. 49, 363001 (2016).

53 R. Shindou, R. Matsumoto, and S. Murakami, "Topological chiral magnonic edge mode in a magnonic crystal”, Phys. Rev. B 87, 174427 (2013).

54 L. F. Zhang, J. Ren, J. S. Wang, and B. W. Li, "Topological magnon insulator in insulating ferromagnet”, Phys. Rev. B 87, 144101 (2013).

$55 \quad$ E. Schlömann, "Generation of spin waves in nonuniform magnetic field. I. Conversion of electromagnetic power into spin-wave power and vice versa", J. Appl. Phys. 35, 159 (1964).

56 E. Schlömann and R. I. Joseph, "Generation of spin waves in nonuniform dc magnetic fields. II. Calculation of the coupling length”, J. Appl. Phys. 33, 167 (1964). 
57 Y. V. Gulyaev, P E. Zilberman, E. S. Sannikov, V. V. Tikhonov, and A. V. Tolkachev, "Linear excitation of pulses of exchange spin-waves in iron-yttrium garnet films", Pis'ma Zh. Tekhn. Fiz. (Leningrad) 14, 884 (1988).

58 Y. I. Gorobets, A. N. Kuchko, and S. V. Vasil'yev, "Excitation of modulated spin waves by model one-dimension anisotropy defect", Fiz. Metall. Metalloved. 85, 40 (1998).

59 C. S. Davies, A. V. Sadovnikov, S. V. Grishin, Y. P. Sharaevskii, S. A. Nikitov, and V. V. Kruglyak, "Generation of propagating spin waves from regions of increased dynamic demagnetising field near magnetic antidots”, Appl. Phys. Lett. 107, 162401 (2015).

$60 \quad$ C. S. Davies and V. V. Kruglyak, "Generation of propagating spin waves from edges of magnetic nanostructures pumped by uniform microwave magnetic field", IEEE Trans. Magn. 52, 2300504 (2016).

61 Y. Au, T. Davison, E. Ahmad, P. S. Keatley, R. J. Hicken, and V. V. Kruglyak, "Excitation of propagating spin waves with global uniform microwave fields", Appl. Phys. Lett. 98, 122506 (2011).

62 Y. Au, E. Ahmad, O. Dmytriiev, M. Dvornik, T. Davison, and V. V. Kruglyak, "Resonant microwave-to-spin-wave transducer", Appl. Phys. Lett. 100, 182404 (2012).

63 M. Arikan, Y. Au, G. Vasile, S. Ingvarsson, and V. V. Kruglyak, "Broadband injection and scattering of spin waves in lossy width-modulated magnonic crystal waveguides", J. Phys. D.: Appl. Phys. 46, 135003 (2013).

64 M. Dvornik, Y. Au, and V. V. Kruglyak, "Micromagnetic simulations in magnonics", Top. Appl. Phys. 125, 101 (2013).

65 N. I. Polushkin, "Combined electron resonance driven by an all-oscillating potential of patterned magnets", Phys. Rev. Lett. 103, 077201 (2009).

66 H. M. Yu, G. Duerr, R. Huber, M. Bahr, T. Schwarze, F. Brandl, and D. Grundler, “Omnidirectional spin-wave nanograting coupler", Nature Comm. 4, 2702 (2013).

67 H. M. Yu, O. d'Allivy Kelly, V. Cros, R. Bernard, P. Bortolotti, A. Anane, F. Brandl, F. Heimbach, and D. Grundler, "Approaching soft X-ray wavelengths in nanomagnet-based microwave technology", Nature Comm. 7, 11255 (2016).

68 S. J. Hermsdoerfer, H. Schultheiss, C. Rausch, S. Schafer, B. Leven, S. K. Kim, and B. Hillebrands, "A spin-wave frequency doubler by domain wall oscillation", Appl. Phys. Lett. 94, 223510 (2009).

69 A. N. Marchenko and V. N. Krivoruchko, "Magnetic structure and resonance properties of a hexagonal lattice of antidots", Low. Temp. Phys. 38, 157 (2012).

$70 \quad$ P. E. Roy, T. Trypiniotis, and C. H. W. Barnes, "Micromagnetic simulations of spinwave normal modes and the resonant field-driven magnetization dynamics of a 360 degrees domain wall in a soft magnetic stripe", Phys. Rev. B 82, 134411 (2010).

71 C. T. Boone and I. N. Krivorotov, "Magnetic domain wall pumping by spin transfer torque”, Phys. Rev. Lett. 104, 167205 (2010).

72 B. Mozooni and J. McCord, "Direct observation of closure domain wall mediated spin waves", Appl. Phys. Lett. 107, 042402 (2015). 
73 B. Van de Wiele, S. J. Hamalainen, P. Balaz, F. Montoncello, and S. van Dijken, "Tunable short-wavelength spin wave excitation from pinned magnetic domain walls", Sci. Rep. 6, 21330 (2016).

74 V. Sluka, M. Weigand, A. Kakay, A. Erbe, V. Tyberkevych, A. Slavin, A. Deac, J. Lindner, J. Fassbender, J. Raabe, and S. Wintz, "Stacked topological spin textures as emitters for multidimensional spin wave modes", Abstract DE-03 in the Book of Abstracts of the 2015 IEEE Intermag Conference (May 11-15, 2015, Beijing, China).

75 J. Truetzschler, K. Sentosun, B. Mozooni, R. Mattheis, and J. McCord, "Magnetic domain wall gratings for magnetization reversal tuning and confined dynamic mode localization", Sci. Rep. 6, 30761 (2016).

76 G. Pöschl and E. Teller, "Bemerkungen zur Quantenmechanik des anharmonischen Oszillators", Z. Phys. 83, 143 (1933).

77 V. E. Demidov, J. Jersch, S. O. Demokritov, K. Rott, P. Krzysteczko, and G. Reiss, "Transformation of propagating spin-wave modes in microscopic waveguides with variable width", Phys. Rev. B 79, 054417 (2009).

78 V. E. Demidov, S. Urazhdin, A. Zholud, A. V. Sadovnikov, and S. O. Demokritov, "Dipolar field-induced spin-wave waveguides for spin-torque magnonics", Appl. Phys. Lett. 106, 022403 (2015).

79 J. Lan, W. C. Yu, R. Q. Wu, and J. Xiao, "Spin-wave diode", Phys. Rev. X 5, 041049 (2015).

80 F. Garcia-Sanchez, P. Borys, R. Soucaille, J. P. Adam, R. L. Stamps, and J. V. Kim, "Narrow Magnonic Waveguides Based on Domain Walls", Phys. Rev. Lett. 114, 247206 (2015).

81 N. Perez and L. Lopez-Diaz, "Magnetic field induced spin-wave energy focusing", Phys. Rev. B 92, 014408 (2015).

82 O. Dzyapko, I. V. Borisenko, V. E. Demidov, W. Pernice, and S. O. Demokritov, "Reconfigurable heat-induced spin wave lenses", Appl. Phys. Lett. 109, 232407 (2016).

83 R. Hertel, W. Wulfhekel, and J. Kirschner, "Domain-wall induced phase shifts in spin waves", Phys. Rev. Lett. 93, 257202 (2004).

84 S. V. Vasiliev, V. V. Kruglyak, M. L. Sokolovskii, and A. N. Kuchko, "Spin wave interferometer employing a local nonuniformity of the effective magnetic field", J. Appl. Phys. 101, 113919 (2007).

85 N. Kanazawa, T. Goto, K. Sekiguchi, A. B. Granovsky, C. A. Ross, H. Takagi, Y. Nakamura, and M. Inoue, "Demonstration of a robust magnonic spin wave interferometer", Sci. Rep. 6, 30268 (2016).

86 K. S. Lee and S. K. Kim, "Conceptual design of spin wave logic gates based on a Mach-Zehnder-type spin wave interferometer for universal logic functions", J. Appl. Phys. 104, 053909 (2008).

87 G. Csaba, A. Papp, and W. Porod, "Spin-wave based realization of optical computing primitives", J. Appl. Phys. 115, 17C741 (2014). 
$88 \quad$ S. Klingler, P. Pirro, T. Bracher, B. Leven, B. Hillebrands, and A. V. Chumak, "Spinwave logic devices based on isotropic forward volume magnetostatic waves", Appl. Phys. Lett. 106, 212406 (2015).

89 F. Gertz, A. Kozhevnikov, Y. Khivintsev, G. Dudko, M. Ranjbar, D. Gutierrez, H. Chiang, Y. Filimonov, and A. Khitun, "Parallel read-out and database search with magnonic holographic memory”, IEEE Trans. Magn. 52, 3401304 (2016).

$90 \quad$ K. Vogt, F. Y. Fradin, J. E. Pearson, T. Sebastian, S. D. Bader, B. Hillebrands, A. Hoffmann, and H. Schultheiss, "Realization of a spin-wave multiplexer", Nature Comm. 5, 3727 (2014).

91 A. V. Sadovnikov, C. S. Davies, S. V. Grishin, V. V. Kruglyak, D. V. Romanenko, Y. P. Sharaevskii, and S. A. Nikitov, "Magnonic beam splitter: The building block of parallel magnonic circuitry", Appl. Phys. Lett. 106, 192406 (2015).

92 C. S. Davies, A. V. Sadovnikov, S. V. Grishin, Y. P. Sharaevskii, S. A. Nikitov, and V. V. Kruglyak, "Field-controlled phase-rectified magnonic multiplexer", IEEE Trans. Magn. 51, 3401904 (2015).

93 T. Braecher, P. Pirro, J. Westermann, T. Sebastian, B. Lagel, B. Van de Wiele, A. Vansteenkiste, and B. Hillebrands, "Generation of propagating backward volume spin waves by phase-sensitive mode conversion in two-dimensional microstructures", Appl. Phys. Lett. 102, 132411 (2013).

94 K. Nanayakkara, A. Anferov, A. P. Jacob, S. J. Allen, and A. Kozhanov, "Cross junction spin wave logic architecture”, IEEE Trans. Magn. 50, 3402204 (2014).

95 Unfortunately, the terminology used in Refs. 26,90,92 was different from that conventionally accepted in the field of electronics. So, we correct it here.

96 A. V. Vashkovsky and E. H. Lock, "Properties of backward electromagnetic waves and negative reflection in ferrite films", Physics-Uspekhi 49, 389 (2006).

97 E. H. Lock, "The properties of isofrequency dependences and the laws of geometrical optics", Physics-Uspekhi 51, 375 (2008).

98 K. R. Smith, M. J. Kabatek, P. Krivosik, and M. Z. Wu, "Spin wave propagation in spatially nonuniform magnetic fields", J. Appl. Phys. 104, 043911 (2008).

99 D. A. Allwood, G. Xiong, C. C. Faulkner, D. Atkinson, D. Petit, and R. P. Cowburn, "Magnetic domain-wall logic", Science 309, 5741 (2005).

100 X. J. Xing, Q. L. Jin, S. W. Li, "Frequency-selective manipulation of spin waves: micromagnetic texture as amplitude valve and mode modulator", New J. Phys. 17, 023020 (2015).

101 D. S. Han, S. K. Kim, J. Y. Lee, S. J. Hermsdoerfer, H. Schultheiss, B. Leven, and B. Hillebrands, "Magnetic domain-wall motion by propagating spin waves", Appl. Phys. Lett. 94, 112502 (2009).

102 V. N. Krivoruchko, "Spin waves damping in nanometre-scale magnetic materials (Review Article)", Low Temp. Phys. 41, 670 (2015).

103 I. Turek, J. Kudrnovsky, and V. Drchal, "Nonlocal torque operators in ab initio theory of the Gilbert damping in random ferromagnetic alloys", Phys. Rev. B 92, 214407 (2015). 
104 M. A. W. Schoen, D. Thonig, M. L. Schneider, T. J. Silva, H. T. Nembach, O. Eriksson, O. Karis, and J. M. Shaw, "Ultra-low magnetic damping of a metallic ferromagnet”, Nature Phys. 12, 839 (2016).

105 J.-N. Toedt, S. Mansfeld, D. Mellem, W. Hansen, D. Heitmann, and S. Mendach, "Interface modes at step edges of media with anisotropic dispersion", Phys. Rev. B 93, 184416 (2016).

106 Y. Au, M. Dvornik, O. Dmytriiev, and V. V. Kruglyak, "Nanoscale spin wave valve and phase shifter", Appl. Phys. Lett. 100, 172408 (2012). 